\title{
CORRECTIONS
}

\section{An ECG that changed in a febrile patient}

This Endgames picture quiz contains an error in the first paragraph (BMJ 2013;346:f585, doi:10.1136/bmj.f585). The last line should have stated that computed tomography of the brain showed a hypodense area in the right frontoparietal area, not the left, as was incorrectly published.
Cite this as: BMJ 2013;346:f981

๑ BMJ Publishing Group Ltd 2013 